\title{
Simple Method for the Elimination of Polarization Noise in BOTDA Using Balanced Detection and Orthogonal Probe Sidebands
}

\author{
Alexia Lopez-Gil, Alejandro Dominguez-Lopez, Sonia Martin-Lopez, and Miguel Gonzalez-Herraez
}

\begin{abstract}
Polarization noise arises in Brillouin optical timedomain analysis due to the strong polarization sensitivity of stimulated Brillouin scattering. To avoid this noise, it turns out to be indispensable to perform some kind of polarization scrambling, either in the pump pulse, the probe signal or both. This is usually achieved using polarization scrambling/switching systems, which, being mechanical, tend to be not as robust as it would be desirable. In this paper, we propose a completely passive system, with no moving parts, to eliminate polarization noise in a BOTDA. It is based on the use of passive depolarization of the pump pulse together with balanced detection among orthogonally polarized Stokes and anti-Stokes bands of the probe signal. The setup requires no alignment readjustment over time and provides a performance similar to a conventional BOTDA using scrambler.
\end{abstract}

Index Terms-Balanced detection, Brillouin scattering, distributed optic fiber sensor, polarization noise, temperature sensor.

\section{INTRODUCTION}

I $\mathrm{N}$ the last years, Brillouin-based distributed temperature and strain sensors have attracted great interest thanks to their unique capabilities to monitor large linear infrastructures. The interest in these systems has been boosted both in the academic and industrial sectors, leading to many demonstrations of the technology in field trials. Specifically, Brillouin optical timedomain analysis (BOTDA) has evolved into a consolidated fiber sensing technology that is widely used in different application domains, such as dams, bridges, railways, pipelines, etc.

The underlying physical phenomenon at the heart of a BOTDA is the optical effect denominated stimulated Brillouin scattering (SBS). SBS is usually described as the interaction of two counter propagating waves, a strong pump wave and a weak probe wave. If particular phase matching conditions are met (namely $f_{\text {Pump }}=f_{\text {probe }}+\nu_{B}, \nu_{B}$ being the Brillouin

Manuscript received September 5, 2014; revised November 11, 2014 and December 10, 2014; accepted December 10, 2014. Date of publication December 17,2014; date of current version April 29, 2015. This work was supported in part by the Spanish Ministry of Science and Innovation through projects TEC201237958-C02-01, TEC2012-37958-C02-02, and TEC2013-45265-R, the Comunidad de Madrid through project FACTOTEM2, SINFOTON-CM: S2013/MIT2790, the INTERREG SUDOE program ECOAL-MGT, and the European Research Council through Starting Grant U-FINE under Grant 307441 and EU-FP7 ITN ICONE. The work of S. Martin-Lopez was supported by the Spanish Ministry of Science and Innovation through a "Ramón y Cajal" contract.

The authors are with the Departamento de Electrónica, Universidad de Alcalá, Edificio Politécnico, Alcalá de Henares, Madrid 28871, Spain (e-mail: alexia.lopez@uah.es; alejandro.dominguez@depeca.uah.es; sonia.martin@ depeca.uah.es; miguelg@ depeca.uah.es).

Color versions of one or more of the figures in this paper are available online at http://ieeexplore.ieee.org.

Digital Object Identifier 10.1109/JLT.2014.2382177 frequency shift), an acoustic wave is generated. This acoustic wave scatters photons from the pump to the probe wave, stimulating the process. From a practical point of view, the process of SBS can be viewed as a narrowband amplification process, in which the pump produces a narrowband $(30-50 \mathrm{MHz})$ gain in a spectral region around $f_{P u m p}-\nu_{B}$ and a loss in the spectral region around $f_{\text {Pump }}+\nu_{B}$. The generation of the acoustic wave in SBS is achieved through the combined action of interference (among the pump and probe waves) and electrostriction [1]. Since interference between pump and probe is indispensable, the efficiency of the SBS process is inherently dependent on the relative states of polarization (SOPs) of the two interacting waves [2]. SBS gain is maximum when the electric fields of the two waves are aligned, i.e., their vectors trace parallel polarization ellipses and in the same sense of rotation. Inversely, if the two ellipses are again similar, but traced in opposite senses of rotation, with their long axes being orthogonal to each other, then the SBS interaction averages to zero over an optical period [3].

BOTDA sensors rely on the distributed measurement of SBS gain along an optical fiber. For that purpose, the pump is pulsed and the probe amplification is analyzed as a function of the time of flight of the pump pulse inside the fiber. Depending on temperature or strain changes, some of the SBS gain attributes (namely the Brillouin frequency shift, BFS) can vary according to well-known sensitivity values. For the SBS gain to occur in a BOTDA, pump and probe waves need to be polarizationaligned, which is usually impossible to guarantee over the full fiber length. In standard single-mode optical fibers, the SOPs of the optical waves change randomly because of random birefringence along the fiber [4]. In this way, if the BOTDA interaction is performed on a conventional fiber, there appear random positions where the gain is maximized together with positions where the gain is rendered close to zero. This random noise-like pattern in the trace is sometimes referred to as "polarization noise," although it remains constant over short periods of time during which the measurement conditions do not vary. However, in order to provide robust measurement conditions along the fiber, this polarization noise has to be eliminated, and to do so, several passive techniques have been proposed. The most used technique is scrambling the polarization state of the pump wave by using a polarization scrambler in order to obtain a pseudo-depolarization of the light; nevertheless, the polarization scrambler needs a passive complicated design to ensure that the scrambled polarization states cover all the possible polarization states evenly. Conventional polarization scramblers 


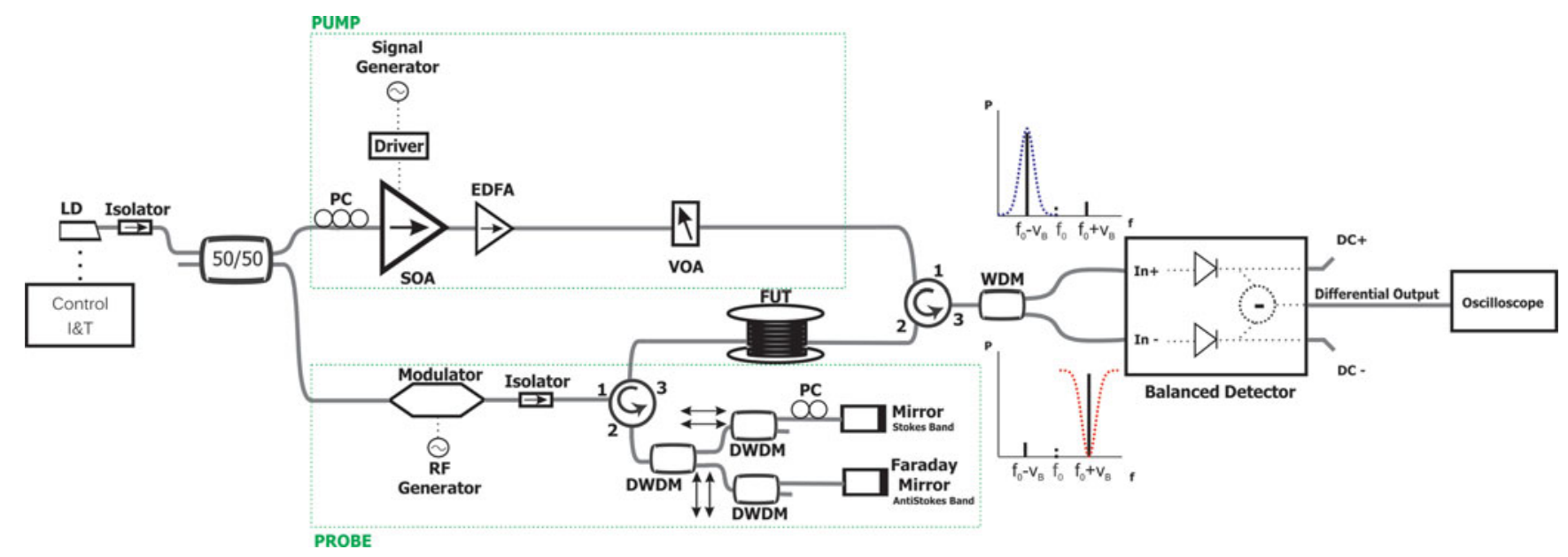

Fig. 1. Experimental setup of the BOTDA with balanced detection and polarization noise elimination. LD: laser diode; PC: polarization controller; EDFA: erbium doped fiber amplifier; RF: radio-frequency generator; VOA: variable optical attenuator; DWDM: dense wavelength division multiplexer.

are typically based on a cascade of electrically controllable fiber squeezers acting as phase retardation plates oriented at different angles (typically there are three stages oriented at $0^{\circ}, 45^{\circ}$ and $90^{\circ}$ ). The three squeezers are activated at three different frequencies so that the SOP at the output along the period of one measurement varies all over the Poincaré sphere in a more or less uniform manner. These fiber squeezers are indeed based on electro-mechanical components and can therefore be thought of as "moving parts." These moving parts make the system less robust than desirable. Some other methods have been developed, such as splitting the pulsed pump into two beams with orthogonal polarization states, one of them delayed by a pulse width, and then recombining them, such that the global system has a resolution of two pulse widths [5], using an unbalanced Mach-Zehnder interferometer in the probe arm [6] or using a polarization switch to get orthogonal polarization states in the probe arm [7]. All of them require moving parts or sophisticated and unstable polarization alignment.

Recently, the use of balanced detection in BOTDA systems was proposed and demonstrated to increase considerably the signal-to-noise ratio (SNR) [8]. This scheme is suitably implemented by using a dual-sideband modulation in the probe wave. Balanced detection among the Stokes and anti-Stokes sidebands in the probe allows a doubling of the trace amplitude and an increase in SNR by a factor of at least $\sqrt{ } 2$ (this analysis is valid when the polarization noise is suppressed using scrambling). In addition, it eliminates common-mode noises in the two sidebands [9].

In this paper, we develop a simple and novel polarization noise elimination method in BOTDA based on the use of balanced detection and orthogonal polarization states in the Stokes and anti-Stokes sidebands [10]. Combined with a depolarization of the pump beam, this method provides comparable results to the method based on polarization scrambler, while being cheaper and more reliable.

\section{EXPERIMENTAL SETUP}

A schematic diagram of the experimental setup used for the tests is depicted in Fig. 1. It is an evolution of a previous BOTDA setup [8] prepared by this group except for the incorporation of a simple set of optical elements to ensure polarization orthogonality of the two probe sidebands. Thus, the detected traces in the positive and negative inputs of the balanced detector are obtained with orthogonal SOPs in the probe wave. Therefore, when there is gain fading on one channel, the other shows maximum gain, and vice versa.

As in most BOTDA schemes, in this setup pump and probe waves are obtained from a single distributed feedback laser diode (1.6 MHz linewidth). A Mach-Zehnder electro-optic modulator (EOM) is used to obtain a probe with two sidebands and a suppressed carrier. The modulating frequency of the EOM is chosen to sweep around the Brillouin frequency shift (BFS) of the fiber under test. The probe sidebands will be either amplified (the lower frequency one - Stokes) or attenuated (the higher frequency one, anti-Stokes) by SBS in the fiber under test. To provide orthogonal SOPs to these two sidebands, a simple scheme based on a circulator, three DWDM filters and a couple of mirrors (one conventional, another one a Faraday mirror) is built. The DWDM filters separate the two side bands of the probe signal.

Three DWDM filters have been used instead of only one to separate both sidebands properly. In the arm of the lower frequency band, a conventional optical mirror is used to reflect the signal without polarization change. On the other side, in the arm of the higher frequency band, a Faraday mirror is used. In this case, the reflected signal polarization is rotated by $90^{\circ}$ with respect to the incident signal. In the port 3 of the optical circulator, we obtain the probe signal composed of the two sidebands (recombined by the DWDM) with orthogonal polarizations.

This method is based on the premise that, with the proposed setup, the two probe sidebands are always launched into the fiber in orthogonal SOP. In the arm of the higher frequency band, thanks to the Faraday mirror, the SOP is rotated exactly $90^{\circ}$ with respect to the incident signal without being dependent on the fiber transfer function. In the arm of the lower frequency band, the back-reflected SOP should be perfectly parallel to the incident one. If the circulator, DWDM and mirrors used in the setup were all PM (as the modulator output and the isolator), 
this condition would be automatically guaranteed. However, using conventional SMF in these components the back-reflected SOP is generally not equal to the incident one. The polarization controller (PC) is then included in the setup to ensure that this condition in the back-reflected field is met. As the length of fiber in this arm is very small (only a few $\mathrm{cm}$ ), the polarization transfer function of this small piece of fiber remains essentially constant over days once the PC is correctly set.

To further illustrate the polarization evolution of the two probe sidebands in this setup, we use now a mathematical model of polarization propagation in these fiber sections by means of Jones matrices [11]. Propagation in a loss-less optical device can be written as a unitary $2 \times 2$ complex matrix called Jones matrix [12], [13]. The input electric field $\overline{E_{i}}$ (defined as a vector with complex components $E_{i x}$ and $E_{i y}$ ) is related to the output electric field $\overline{E_{o}}$ as $\overline{E_{o}}=U \bar{E}_{i}$, where

$$
U=\left[\begin{array}{ll}
u_{1} & u_{2} \\
-u_{2}^{*} & u_{1}^{*}
\end{array}\right]
$$

with $\left|u_{1}\right|^{2}+\left|u_{2}\right|^{2}=1$ (for detailed information on how the matrix $U$ is constructed, the interested reader may use reference [11]).

In the following, we fix the reference frame so that its $z$-axis coincides with forward direction [14].

The Jones matrix of backward propagation is related to the Jones matrix of forward propagation by the following relationship [13]:

$$
\overleftarrow{U}=\vec{U}^{T}
$$

The matrix representing the round-trip in each arm (fiber in forward direction, mirror/Faraday mirror $-M$-, fiber in backward direction) is easily obtained

$$
U_{B}=\overleftarrow{U} M \vec{U}=\vec{U}^{T} M U
$$

where the matrix $M$ is the identity for a conventional mirror [14]. The matrix representing the Faraday mirror can be written as [15]:

$$
M=\left[\begin{array}{cc}
0 & -1 \\
1 & 0
\end{array}\right] .
$$

We can now compute the value of $U_{B}$ in the case of the Faraday mirror arm to find:

$$
U_{B}=\left[\begin{array}{cc}
0 & -1 \\
1 & 0
\end{array}\right]
$$

which is exactly the same as that of the Faraday mirror. Thus, in this case, the back-reflected SOP appears always rotated $90^{\circ}$ with respect to the incident signal without dependence on the Jones matrix of the optical fiber in the middle.

On the other hand, in the arm of the lower frequency band, we would need that $U_{B}$ equals the identity matrix (possibly with a change in the global phase of the field) to achieve the same polarization state as the input. It can be shown that this does not generally occur except in media exhibiting no birefringence at all or pure circular birefringence. As this is not generally satisfied in conventional optical fibers, it is necessary to insert a PC in this arm. In our experimental setup this arm is kept as short as possible (a few $\mathrm{cm}$ ) to avoid random polarization evolution due to varying ambient conditions. We note again that the condition required for this arm (reflection parallel to the input field) would be satisfied for PM components and a deterministic polarization state at the input.

A possible evolution of this setup would be then to have these components (isolator, circulator, DWDM and mirrors in the probe arm) in PM fiber. This would ensure perfect polarization orthogonality in all cases without any PC alignment.

With the described setup, the polarization scrambler used in [8] is no longer necessary to eliminate the dependence of the Brillouin gain on polarization. The probe power fed into the fiber is $\sim 330 \mu \mathrm{W}$ on each sideband.

At the pump side, a semiconductor optical amplifier (SOA) is used to shape the pulse. The pulse width is varied in the range from 20 to $50 \mathrm{~ns}$ and the repetition rate is fixed to $1.6 \mathrm{KHz}$. The pulses are then amplified through an erbium doped fiber amplifier (EDFA) and the output power is controlled using a variable optical attenuator (VOA). The peak power of the pulses fed into the fiber is several tens of $\mathrm{mW}$. A high-speed MEMS switch is inserted in the scheme to improve the extinction ratio of the pulses obtained at the output of the SOA $(\sim 40 \mathrm{~dB})$.

After going through the fiber and experiencing Brillouin scattering, the probe sidebands are then separated using a conventional DWDM filter, which splits Brillouin gain band (Stokes) and Brillouin loss band (anti-Stokes). The edge of the filter used is sharp enough to split both sidebands correctly, with a rejection of the opposite band of $>13 \mathrm{~dB}$. These two different bands are then fed into the positive and negative ports of the balanced detection system. In our case, the differential output will be the result of subtracting the negative input signal (anti-Stokes band) to the positive input signal (Stokes band). In terms of the polarization noise, since each trace is measured with an orthogonal SOP, the maxima of polarization noise on one trace match with the minima of the polarization noise of the other one. Overall, the trace obtained in the balanced output shows a polarization noise that is mostly removed. Ultimately, the balanced detection system provides three different output signals: the differential output (with the signal of interest), and two monitoring outputs, where we will read the dc levels of each of the input signals. The sum of these dc levels is used to normalize the detected trace and obtain gain measurements.

\section{Elimination of Polarization Noise}

In this section, we illustrate the results obtained with the configuration described previously in terms of elimination of polarization noise. The measurements have been performed using a Brillouin pump pulse-width of $20 \mathrm{~ns}$ ( $2 \mathrm{~m}$ spatial resolution) over $\sim 50 \mathrm{~km}$ of single-mode fiber (composed of two different fiber spools of $25 \mathrm{~km}$ each one spliced together). The fiber has an effective area of $70 \mu \mathrm{m}^{2}$ and an essentially homogeneous BFS located at approximately $10.883 \mathrm{GHz}$ at the pump wavelength $(\sim 1550 \mathrm{~nm})$. To achieve a clean trace, the traces are averaged 

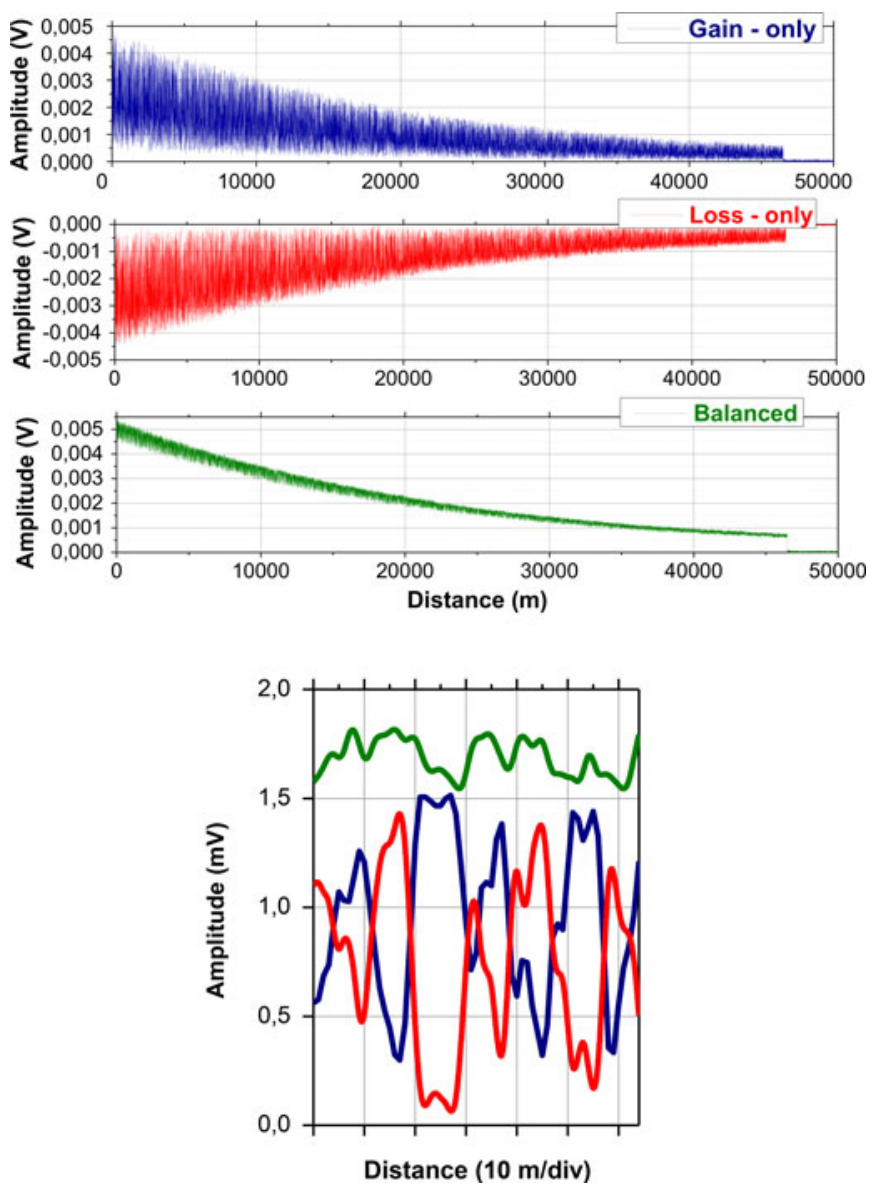

Fig. 2. (a) Trace obtained using the single-detector cases (two on top) and balanced detection (one on bottom) for a pump-probe frequency shift of $10.883 \mathrm{GHz}$. (b) A detail of $64 \mathrm{~m}$ of the three traces of Fig (a) around $25 \mathrm{~km}$.

1024 times. In Fig. 2 the three traces obtained at $10.883 \mathrm{GHz}$ for the single sideband detection cases (the two on top) and balanced detection (the one on bottom) are shown. Fig. 2(b) shows a detail of $64 \mathrm{~m}$ of the three traces of Fig. 2(a) around $25 \mathrm{~km}$. It can be observed that the maxima and the minima of the blue (gain) and red (attenuation) traces are located at the same position. Therefore the polarization noise in the balanced channel is mostly removed. This is an expected result considering the explanation given in Section II. Moreover, as is described in [8], when acquiring in balanced mode the trace amplitude of the differential output is twice the trace amplitude of any the single bands and the robustness of the system increases to common-mode noises, which affect both sidebands. For these reasons, the SNR of this scheme is considerably higher than a single-detector system.

Fig. 3 shows the gain profile as a function of the probe frequency at a distance of $48.5 \mathrm{~km}$, with a frequency sweep done from 10.78 to $10.98 \mathrm{GHz}$ for the balanced detection acquisition. As it can be inferred, the Brillouin full-width at half maximum is approximately $35 \mathrm{MHz}$. No major broadening or deformation of the gain profile is observed, which seems to indicate that the measurement system is performing correctly.

As it is noticeable, a perfect polarization noise cancellation has not been achieved in the trace. We measured the polarization

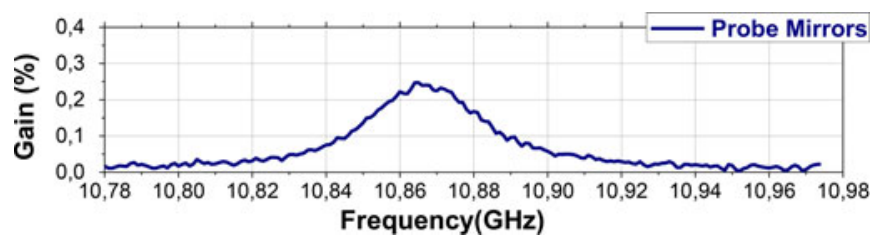

Fig. 3. Gain profile at $48.5 \mathrm{~km}$ for a frequency sweep between 10.78 and $10.98 \mathrm{GHz}$ using balanced detection and orthogonal polarizations in the Stokes and anti-Stokes bands.

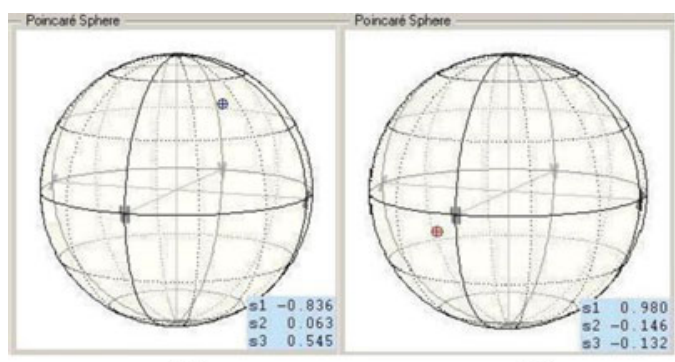

(a)

(b)

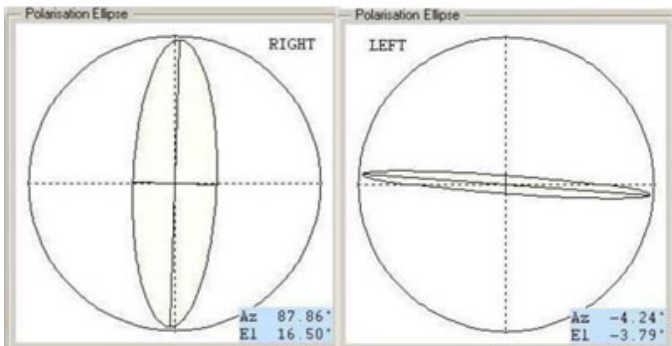

(c)

(d)

Fig. 4. Polarization state displayed in the Poincarè Sphere of Brillouin Stokes band (a) and Brillouin anti-Stokes band (b), and displayed in the Polarization Ellipse for the Brillouin Stokes band (c) and Brillouin anti-Stokes band (d).

states of the two sidebands at the fiber input and they were not perfectly orthogonal, although the disagreement was not excessive (see Fig. 4). This is probably due to some manufacturing imperfections of the Faraday mirror. In addition, a small mismatch in the optical path lengths of the two sidebands, and any small error in the adjustment of the laser could lead to a nonsymmetric separation of the two bands, causing an imperfect polarization noise cancellation.

\section{Performance in Terms of BFS Determination}

In this section, we illustrate the results obtained with the configuration described previously in terms of BFS determination. Again, the probe frequency was swept over the range of interest and the resulting curve was fitted using the standard procedures. Repeatability among five consecutive sweeps was evaluated by computing the standard deviation of the BFS results. The same process is done in the conventional balanced detection setup incorporating a high-performance scrambler (model Agiltron P/N: NOPS-123111331, with a scrambling frequency of $5 \mathrm{MHz}$ and operating wavelength range of $1300-1600 \mathrm{~nm}$ ) [8]. In order to evaluate only the uncertainty caused by the measurement method, the pulse length was increased up to $50 \mathrm{~ns}$ to ensure the best possible measurement conditions in the standard 


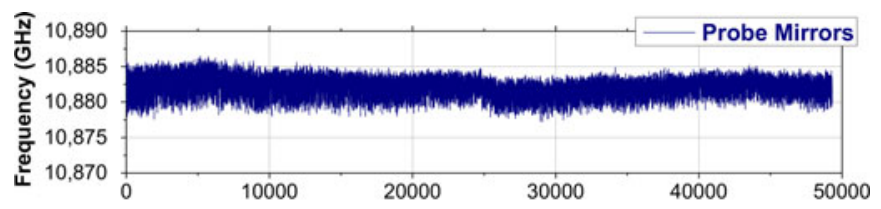

(a)

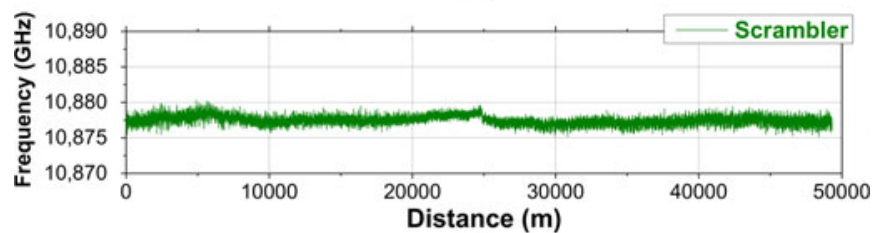

(b)

Fig. 5. BFS of the balanced detection acquisition for the case with mirrors in the probe arm (a) and the scrambler case (b).

configuration. In both cases, the measurements have been performed using a Brillouin pump pulse-width of $50 \mathrm{~ns}$ ( $5 \mathrm{~m}$ spatial resolution) over the same $50 \mathrm{~km}$ of single mode fiber (SMF) as in the previous test. The same amount of averages is used in all cases (1024) so as to ensure a fair comparison. The probe power fed into the fiber is $\sim 320 \mu \mathrm{W}$ on each sideband and the pulse peak power fed into the fiber is $\sim 18 \mathrm{~mW}$. The BFS determination results are plotted in Fig. 5(a) and (b). As it is visible, in the scrambler case, the BFS determination seems to have been done with less uncertainty unlike the probe depolarization case in which the results seem to be slightly worse. What is more intriguing is that the BFS uncertainty in the probe depolarization case seems to be larger at the beginning than at the end of the fiber, unlike the conventional case where the uncertainty increases towards the end. In terms of numbers, the standard deviation of the BFS computed over a 5000-point window is $1.6 \mathrm{MHz}$ at the beginning of the fiber and $1.1 \mathrm{MHz}$ at the end, while it is only $0.6 \mathrm{MHz}$ in the worst case of the scrambler (fiber end). The origin of this extra uncertainty in the proposed configuration is quite unclear and the authors are trying to put forward a convincing explanation on why such behavior happens. However, it is clear that scrambling the pump leads to a complete cancellation of this effect.

\section{IMPROVEMENT USING PUMP DEPOLARIZATION}

In order to avoid the problems explained in the previous section, it seems convenient to scramble or at least depolarize the pump to avoid the extra uncertainty given by this issue. To do so, we additionally developed a pump de-polarization method over the previous experiment, for which the schematic diagram is depicted in Fig. 6 (only the pump branch is shown, as the rest of the scheme remains as in Fig. 1).

In this case we benefit from a polarization-insensitive SOA, with a polarization dependent gain of $<1 \mathrm{~dB}$. Before shaping the pump pulse using the SOA, a passive depolarization of the pump light is provided using a simple scheme based on a circulator, a 50/50 optical coupler, a $200 \mathrm{~m}$ optical fiber spool and a couple of mirrors (one conventional, and another one a Faraday mirror). The length of optical fiber inserted between the coupler and the mirror is chosen to be longer than the coherence length of the

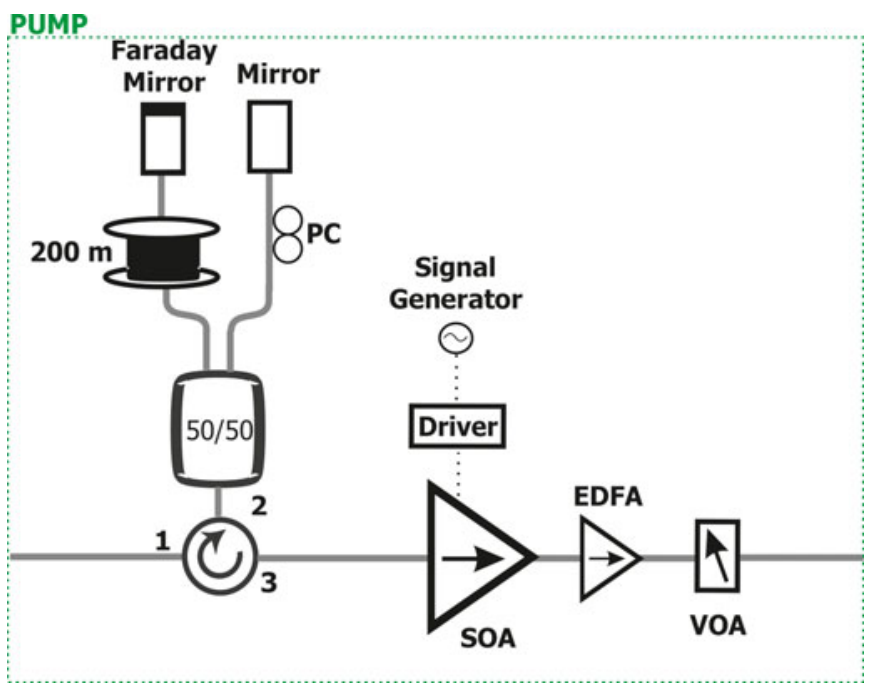

Fig. 6. Schematic of the pump arm of the BOTDA with balanced detection and polarization noise elimination. PC: polarization controller; EDFA: erbium doped fiber amplifier; VOA: variable optical attenuator.

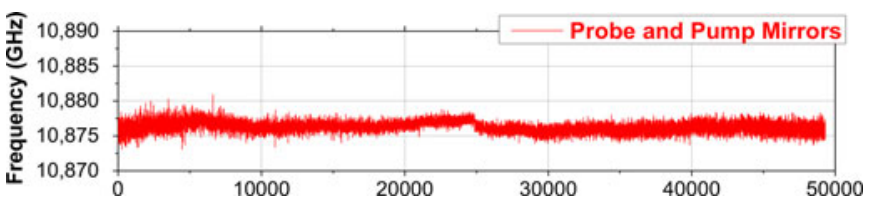

Fig. 7. BFS of the balanced detection acquisition for the case with mirrors in the probe and pump arms.

TABLE I

STANDARD DEVIATION OF THE OBTAINED BFS

\begin{tabular}{lcc}
\hline \hline Scheme & Beginning of the fiber & End of the fiber \\
\hline Probe Mirrors & 1.6 & 1.1 \\
Probe and Pump Mirrors & 0.6 & 0.8 \\
Scrambler & 0.4 & 0.6 \\
\hline \hline
\end{tabular}

laser. Again, it should be stressed that the length of fiber in the conventional mirror arm is kept as short as possible.

Again, the obtained traces show a well-suppressed polarization noise. Moreover, in Fig. 7, the BFS as a function of the distance is obtained exactly with the same methodology and settings as in the previous section.

A comparison of the standard deviations of BFS is depicted in the Table I. The standard deviation of the obtained BFS among five consecutive traces at the beginning of the fiber (first 5000 points window) is $\sim 1.6 \mathrm{MHz}$ for the case with mirrors in the probe arm, $\sim 0.6 \mathrm{MHz}$ for the case with mirrors in the probe and pump arms and $\sim 0.4 \mathrm{MHz}$ for the scrambler case. At the end of the fiber under test (last 5000 points window), the standard deviation is $\sim 1.1 \mathrm{MHz}$ for the case with mirrors in the probe arm, $\sim 0.8 \mathrm{MHz}$ for the case with mirrors in the probe and pump arms and $\sim 0.6 \mathrm{MHz}$ for the scrambler case. As illustrated by these results, the setup including pump depolarization and probe mirrors is nearly as effective in removing polarization noise as a commercial polarization scrambler with nearly the same 
performance in terms of BFS determination. Having no moving part and being much cheaper, we believe that this solution is certainly advantageous over the traditional polarization scrambler.

\section{CONCLUSION}

In this paper we have presented a method to avoid the polarization noise in BOTDA systems. Our method is based on the balanced detection of traces obtained with orthogonally polarized Stokes and anti-Stokes sidebands. Our scheme has no moving parts and provides clear improvements in terms of cost. As all the systems based on balanced detection, our system is also robust to common-mode noises.

A slightly larger uncertainty in terms of BFS determination is obtained with the proposed method. In order to avoid this problem we propose another scheme (with no moving parts) which performs polarization scrambling also in the pump pulse. In terms of performance, this new system provides results comparable to the conventional systems employing scramblers. Considering the increase in robustness and the reduced price, our option looks appealing for integration in field systems.

A problem of reduced quality in our method may arise as a result of a fiber section with high PMD. Indeed, a high PMD section in the measurement fiber could lead to a significant loss in the orthogonality of the SOPs of the two probe sidebands, and therefore to a significant growth of polarization noise thereafter. However, given the high values of PMD required to observe this problem (differential group delays of several ps), it should not be a drawback using modern optical fibers with low PMD values.

It should also be noted that any element with significant polarization dependent loss in the probe path may also lead to a significant loss of performance of the setup in terms of polarization noise elimination.

\section{ACKNOWLEDGMENT}

The authors would like to thank insightful discussions with Profs. L. Thévenaz and M. Tur.

\section{REFERENCES}

[1] G. P. Agrawal, Nonlinear Fiber Optics, 4th ed. San Diego, CA, USA: Academic, 2007, ch. 9.

[2] R. H. Stolen, "Polarization effects in fiber Raman and Brillouin lasers," IEEE J. Quantum Electron., vol. 15, no. 10, pp. 1157-1160, Oct. 1979.

[3] A. Zadok, E. Zilka, A. Eyal, L. Thévenaz, and M. Tur, "Vector analysis of stimulated Brillouin scattering amplification in standard single-mode fibers," Opt. Exp., vol. 16, no. 26, pp. 21692-21707, 2008.

[4] M. O. Van Deventer, and A. J. Boot, "Polarization properties of stimulated Brillouin scattering in single-mode fibers," J. Lightw. Technol., vol. 12 no. 4, pp. 585-590, Apr. 1994.

[5] J. Yang, C. Yu, Z. Chen, J. Ng, and X. Yang, "Suppression of polarization sensitivity in BOTDA fiber distributed sensing system," Proc. SPIE, vol. 7004, p. 700421, 2008.

[6] S. Diaz, S. F. Mafang, M. Lopez-Amo, and L. Thevenaz, "A highperformance optical time-domain Brillouin distributed fiber sensor," IEE Sens. J., vol. 8, no. 7, pp. 1268-1272, 2008.

[7] M. A. Soto, X. Angulo-Vinuesa, S. Martin-Lopez, S. H. Chin, J. D. Ania-Castañon, P. Corredera, E. Rochat, M. Gonzalez-Herraez, and L. Thévenaz, "Extending the real remoteness of long-range Brillouin optical time-domain fiber analyzers," J. Lightw. Technol., vol. 32, no. 1, pp. 152-162, Jan. 2014.

[8] A. Domínguez-López, A. López-Gil, S. Martín-López, and M. González-Herráez, "Signal-to-noise ratio improvement in BOTDA using balanced detection," IEEE Photon. Technol. Lett., vol. 26, no. 4, pp. 338-341, Feb. 2014.

[9] A. Domínguez-López, A. López-Gil, S. Martín-López, and M. GonzálezHerráez, "Strong cancellation of RIN transfer in a Raman-assisted BOTDA using balanced detection," IEEE Photon. Technol. Lett., vol. 26, no. 18, pp. 1817-1820, Sep. 2014

[10] A. Lopez-Gil, A. Dominguez-Lopez, S. Martin-Lopez, and M. GonzalezHerraez, "Simple method for the elimination of polarization noise in BOTDA using balanced detection of orthogonally polarized stokes and anti-stokes probe sidebands," Proc. SPIE, vol. 9157, p. 91573U, 2014

[11] F. Corsi, A. Galtarossa, and L. Palmieri, "Polarization mode dispersion characterization of a single-mode optical fiber using backscattering technique," J. Lightw. Technol., vol. 16, no. 10, pp. 1832-1843, Oct. 1998.

[12] F. Curti, B. Daino, G. De Mar.is, and F. Matera, "Statistical treatment of the evolution of the principal states of polarization in single-mode fibers," J. Lightw. Technol., vol. 8, no. 8, pp. 1162-1166, Aug. 1990.

[13] R. C. Jones, "A new calculus for the treatment of optical system," J. Opt. Soc. Amer, vol. 37, pp. 107-110, 1947.

[14] E. Collett, Ed., Polarized Light Fundamentals and Applications. New York, NY, USA: Marcel Dekker, 1993.

[15] M. Wuilpart and M. Tur, "Polarization effects in optical fibers," in $A d$ vanced Fiber Optics, L. Thévenaz Ed., 1st ed. Lausanne, Switzerland: EPFL Press, 2011, ch., pp. 29-86.

Alexia Lopez-Gil received the telecommunication engineering degree from the Polytechnic University of Madrid, Madrid, Spain, in 2012 and the Master's degree in advanced electronics systems from the University of Alcala, Madrid, Spain, in 2014. Since 2013, she has been working toward the Ph.D. degree in the Photonics Engineering Group, University of Alcala, Madrid, Spain.

Her current research interests include nonlinear fiber optics and fiber optic sensors.

Alejandro Dominguez-Lopez was born in Granada, Spain, in 1987. He received the telecommunication engineering degree from the University of Granada, Granada, Spain, in 2012 and the Master's degree in advanced electronics systems from the University of Alcala, Madrid, Spain, in 2014. Since 2013, he has been working toward the Ph.D. degree in the Photonics Engineering Group, University of Alcala, Madrid, Spain.

His current research interests include nonlinear fiber optics and fiber optic sensors.

Sonia Martin-Lopez received the Ph.D. degree from the Universidad Complutense de Madrid, 28040 Madrid, Spain, in May 2006. The topic of her doctoral dissertation was on experimental and theoretical understanding of continuouswave pumped supercontinuum generation in optical fibers.

She is author or coauthor of more than 100 papers in international refereed journals and conference contribution. Her current research interests include nonlinear fiber optics and optical fiber sensors. She had a stay in the Nanophotonics and Metrology Laboratory, Ecole Polytechnique Federale de Lausanne, Switzerland. She has been engaged as a Postdoctoral Researcher in the Applied Physics Institute and in the Optics Institute at the Spanish Council for Research. She is currently engaged as Postdoctoral Ramon y Cajal Researcher in the Electronic Department of Alcala University of Madrid.

Miguel Gonzalez-Herraez received the M.Eng. and D.Eng. degrees from the Polytechnic University of Madrid, Madrid, Spain, in 2000 and 2004, respectively. While working toward the D.Eng. degree, he worked first as a Research Assistant and then a Postdoctoral Fellow in the Applied Physics Institute at the Spanish Council for Research, Madrid, Spain, and had several long stays in the Nanophotonics and Metrology Laboratory, Ecole Polytechnique Federale de Lausanne, Switzerland. In October 2004, he was appointed as an Assistant Professor in the Department of Electronics, University of Alcalá, Madrid, Spain, where he was promoted to an Associate Professor in June 2006. He is the author or coauthor of more than 160 papers in international refereed journals and conference contributions and has given several invited talks at international conferences. His research interests include the wide field of nonlinear interactions in optical fibers.

Dr. González-Herráez has received several important recognitions to his research career, including the European Research Council Starting Grant and the "Agustin de Betancourt" prize of the Spanish Royal Academy of Engineering. 